\title{
A Partial regularity result for a class of Stationary Yang-Mills Fields in high dimension
}

Yves Meyer and Tristan Rivière

\begin{abstract}
We prove, for arbitrary dimension of the base $n \geq 4$, that stationary Yang-Mills Fields satisfying some approximability property are regular apart from a closed subset of the base having zero $(n-4)$ Hausdorff measure.
\end{abstract}

\section{Introduction}

Let $(M, g)$ be a $n$-dimensional Riemanian Manifold and $E$ a $k$-real vector bundle over $M$ modeled on a principal bundle $Q$ whose structure group $G \subset S O(k)$. Denote $<,>$ the scalar product on $q$-forms into the adjoint bundle ad $E$ which is compatible with the usual metric on $S O(k)$ (the adjoint bundle ad $E$ is issued from $Q$ and the adjoint action of $G$ on its Lie algebra $\mathfrak{G})$. Following [14] we denote

$$
\begin{gathered}
\mathfrak{U}_{k}^{p}=\left\{D=D_{0}+A \quad \text { where } A \in \Omega^{1}(\operatorname{ad} E):=\Gamma\left(M ; \wedge^{1} M \otimes \operatorname{ad} E\right)\right. \\
\text { s.t. } \left.\|A\|_{W^{k, p}(M, a d E)}<+\infty\right\},
\end{gathered}
$$

where $W^{k, p}$ is the usual Sobolev spaces of maps having $l$-th derivatives in $L^{p}$ for $l \leq k$ and $D_{0}$ is a smooth connection of $E\left(\mathfrak{U}_{k}^{p}\right.$ is independent of the choice of $D_{0}$ ).

On $\mathfrak{U}_{1}^{2} \cap \mathfrak{U}_{0}^{4}$ we may consider the Yang-Mills Functional

$$
Y M(D)=\int_{M}<F_{D}, F_{D}>d v o l_{M}
$$

where $F_{D}$ is the curvature of $D$, belonging to $\Omega^{2}(a d E):=\Gamma\left(M ; \wedge^{2} M \otimes a d E\right)$ verifying $F_{D}=F_{D_{0}}+D_{0} A+[A, A]$ where $F_{D_{0}}$ is the curvature of $D_{0}$.

2000 Mathematics Subject Classification: 35D10, 35J20, 35J60, 35Q40, 46Exx, 53C07, 58E15, 81T13.

Keywords: Yang-Mills Fields, Gauge Theory, Non-linear elliptic regularity theory, Interpolation inequalities, Morrey Spaces. 
We also denote by $D_{0}$ the induced connection modeled on $D_{0}$ and acting from $\Omega^{q}(a d E)$ into $\Omega^{q+1}(a d E)$. Finaly [, ] is the bracket on $\Omega^{q}(a d E) \otimes$ $\Omega^{r}(a d E)$ (for complete definitions see for instance [4]). From the formula above one easily verify that $Y M$ is well defined on $\mathfrak{U}_{1}^{2} \cap \mathfrak{U}_{0}^{4}$.

Definition 1.1 A weak Yang-Mills Field is a critical point D in $\mathfrak{U}_{1}^{2} \cap \mathfrak{U}_{0}^{4}$ of the Yang-Mills Functional YM for perturbations of the form $D+$ ta for any $a \in W^{1,2}(M ; a d E) \cap L^{4}(M ; a d E)$. Such a connection is a weak solution of the Yang-Mills equation

$$
D * F_{D}=D_{0}\left(* F_{D}\right)+\left[A, * F_{D}\right]=0 \quad \text { in } \mathcal{D}^{\prime}(M)
$$

where $*$ is the Hodge operator on forms deduced from the metric $g$ on $M$.

Observe that standard computations can be extended to this weak setting and that any connection $D$ in $\mathfrak{U}_{1}^{2} \cap \mathfrak{U}_{0}^{4}$ solves the Bianchi identity

$$
D F_{D}=D_{0}\left(F_{D}\right)+\left[A, F_{D}\right]=0 \quad \text { in } \mathcal{D}^{\prime}(M)
$$

It is proved in [14] that in dimension less or equal to 4 any weak Yang-Mills Field is analytic an therefore solves Yang-Mills Equation (1.1) in a strong sense. This result is not true in higher dimension and one may expect weak Yang-Mills Fields to be singular somewhere (see examples in [13]). It is not excluded that like for harmonic maps they can have large singular set.

Similarly to harmonic maps again and following suggestions in [13] we may consider a subclass among weak Yang-Mills Fields which is made of so called Stationary Yang-Mills Fields which are weak Yang-Mills Fields which are also critical points of $Y M$ for perturbations on $M$. Precisely one introduces the following definition.

Definition 1.2 A connection D in $\mathfrak{U}_{1}^{2} \cap \mathfrak{U}_{0}^{4}$ is a stationary Yang-Mills Fields if it is weak Yang-Mills and if it is also a critical point of YM for the following perturbations

$$
\text { for all } X \in \Gamma(T M) \quad \frac{d}{d t} \int_{M}<\psi_{t}^{*} F_{D}, \psi_{t}^{*} F_{D}>\left.\right|_{t=0}=0
$$

where $\psi_{t}=\exp _{x}(t X)$. Such a Field is a solution of the stationary Yang-Mills Equation (see [11] and [13])

$\forall X \in \gamma(T M) \quad \int_{M}<F_{D}, F_{D}>\operatorname{div} X-4<F_{D}(\nabla X, \cdot), F_{D}>\operatorname{dvol}_{M}=0$ where, using an orthonormal basis $\left(e_{i}\right)_{i=1, \ldots, n}$ of $T_{x} M,\left\langle F_{D}(\nabla X, \cdot), F_{D}>:=\right.$ $\sum_{i, j=1}^{n}<F_{D}\left(\nabla_{e_{i}} X, e_{j}\right), F_{D}\left(e_{i}, e_{j}\right)>$ and $\nabla$ denotes the Levi-Civita connection on $(M, g)$. 
As a consequence of the Stationary Yang-Mills Equation (1.3) one obtains the monotonicity formula (see [11] and Theorem 2.1.2 of [13]) that, in the particular case where $g$ is flat $:(M, g)=\mathbb{R}^{n}$, reads $\forall x \in \mathbb{R}$ and $\forall r>0$

$$
\begin{gathered}
\forall x \in \mathbb{R} \text { and } \forall r>0 \quad \frac{d}{d r}\left[\frac{1}{r^{n-4}} \int_{B_{r}(x)}<F_{D}, F_{D}>d x\right] \\
=\frac{4}{r^{n-4}} \int_{\partial B_{r}(x)}<\iota_{\frac{\partial}{\partial r}} F_{D}, \iota_{\frac{\partial}{\partial r}} F_{D}>d v o l_{\partial B_{r}(x)} \geq 0
\end{gathered}
$$

where $\iota_{\frac{\partial}{\partial r}} F_{D}$ denotes the element of $\Omega^{1}(a d E)$ obtained by contracting the 2form $F_{D}$ and the vector $\frac{\partial}{\partial r}$. Then, if $D$ is a Stationary Yang-Mills Fields and $(M, g)$ has bounded geometry, one gets the following control of the conformal invariant $Y M$-energy density

$$
\sup _{x \in M, r>0} \frac{1}{r^{n-4}} \int_{B_{r}(x)}<F_{D}, F_{D}>d v o l_{M} \leq C \int_{M}<F_{D}, F_{D}>d v o l_{M}
$$

Therefore it is natural to introduce the space of connections $D \in \mathfrak{U}_{1}^{2} \cap \mathfrak{U}_{0}^{4}$ $D=D_{0}+A$ such that

$$
\sup _{x \in M, r>0} \frac{1}{r^{n-4}} \int_{B_{r}(x)}|\nabla A|^{2}+|A|^{4} d^{2} o_{M}<+\infty .
$$

We will adopt the following notation :

$$
\begin{gathered}
\mathfrak{M}_{k}^{l, p}=\left\{D=D_{0}+A \quad \text { where } A \in \Omega^{1}(a d E):=\Gamma\left(M ; \wedge^{1} M \otimes a d E\right)\right. \\
\text { s.t. } \left.\|A\|_{M_{k}^{l, p}(M, a d E)}<+\infty\right\}
\end{gathered}
$$

where $M_{k}^{l, p}$ denotes the following Morrey space

$$
\|u\|_{M_{k}^{l, p}\left(B_{1}, V\right)}=\sup _{x \in B_{1}, r>0}\left(\frac{1}{r^{n-p k}} \int_{B_{1} \cap B_{r}(x)}\left|\nabla^{l} u\right|^{p} d x\right)^{1 / p}
$$

and where $\nabla A$ is the following section in $\Gamma\left(M, T M \otimes \wedge^{1} M \otimes \operatorname{ad} E\right) \nabla A=$ $\sum_{k=1}^{n} e_{k} \otimes\left(D_{0}\right)_{e_{k}} A$ and $e_{k}$ is an orthonormal basis of $T_{x} M$ so that $|\nabla A|^{2}=$ $\sum_{k=1}^{n}\left|\left(D_{0}\right)_{e_{k}} A\right|^{2}$. (We also adopt the notation $|B|^{2}:=<B, B>$ ).

One of the difficulty of working with the above Morrey spaces, although they seem quite relevant to stationary Yang-Mills in high dimension, is the lack of density of connections as long as $n>p k$. This lack of density is the natural counterpart of the possibility (and there are examples) for our stationary Yang-Mills configurations to be singular somewhere. In order to 
compensate this lack of density we will assume the following approximability property for $D$ in $\mathfrak{M}_{2}^{1,2} \cap \mathfrak{M}_{1}^{0,4}$ :

$$
\begin{aligned}
& \forall \varepsilon>0 \quad \exists \alpha>0 \text { s.t. } \forall x_{0} \in M \text { and } \forall 1>r_{0}>0 \\
& \text { if } \sup _{x \in B_{r_{0}}\left(x_{0}\right), r_{0}>r>0} \frac{1}{r^{n-4}} \int_{B_{r}(x)}<F_{D}, F_{D}>d v o l_{M} \leq \alpha
\end{aligned}
$$

then there exists a smooth $D_{i}$ s.t. $D_{i} \rightarrow D$ in $\mathfrak{U}_{1}^{2} \cap \mathfrak{U}_{0}^{4}\left(B_{1}\right)$

$$
\text { such that } \sup _{x \in B_{r_{0} / 2}\left(x_{0}\right), \frac{r_{0}}{2}>r>0} \frac{1}{r^{n-4}} \int_{B_{r}(x)}<F_{D_{i}}, F_{D_{i}}>d v o l_{M} \leq \varepsilon
$$

Any connection $D$ in $\mathfrak{M}_{2}^{1,2} \cap \mathfrak{M}_{1}^{0,4}$ satisfying (1.7) is called "approximable".

Our main result is the following.

Theorem 1.1 Let $D$ be an approximable stationary Yang-Mills connection in $\mathfrak{M}_{2}^{1,2} \cap \mathfrak{M}_{1}^{0,4}$, then there exists a closed subset $\Sigma$ of $M$ such that $\mathcal{H}^{n-4}(\Sigma)=$ 0 and for any $x \in M \backslash \Sigma$ there exists an open neighborhood $U$ of $x$ in $M \backslash \Sigma$ and a trivialization of $Q$ over $U, s$ in $W^{1,4}(U, Q) \cap W^{2,2}(U, Q)$ such that $F_{D}$ expressed in this trivialization is smooth.

The approximability assumption should hold for any stationary Yang-Mills in $\mathfrak{M}_{2}^{1,2} \cap \mathfrak{M}_{1}^{0,4}$ and should be removed from the hypothesis of our theorem. At this stage of the understanding of weak connections, the need of the approximability assumption can be illustrated as follows : Assume a 1-form $A$ in $M_{2}^{1,2}\left(B_{1}^{n}\right) \cap M_{1}^{0,4}\left(B_{1}^{n}\right)$ has zero curvature $d A+[A, A]=0$, it is not known, without the approximability assumption, for $n>4$, whether $A$ is gauge equivalent to the trivial connection or not.

Our partial regularity result has to be compared with the coresponding partial regularity result for harmonic maps into symmetric spaces established by L.C. Evans in [5] (see also Hélein's book [8]). It is a consequence of the following epsilon regularity theorem :

Theorem 1.2 Let $D$ be an approximable Stationary Yang-Mills connection in $\mathfrak{M}_{2}^{1,2} \cap \mathfrak{M}_{1}^{0,4}$, let $K$ be a compact subset of $M$, then there exist positive constants $\varepsilon$ and $C$ such that for any $\rho<1$ and any $x \in K$,

$$
\text { if } \rho^{4-n} \int_{B_{\rho}(x)}\left|F_{D}\right|^{2} \leq \varepsilon, \quad \text { then }\left|F_{D}\right|(x) \leq \frac{C}{\rho^{2}}\left(\rho^{4-n} \int_{B_{\rho}(x)}\left|F_{D}\right|^{2}\right)^{1 / 2} .
$$

This epsilon regularity theorem is a well known fact established in [10] and [13] as long as $D$ is assumed to be a smooth Yang-Mills connection. 
In that case the proof is based on the use of the Weitzenböck-Bochner Formula for smooth Yang-Mills Fields (see [2] for Weitzenböck-Bochner Formula in this context). The difficulty in the present paper was to extend the epsilon regularity to a-priori non smooth Yang-Mills connections for which such a formula does not a-priori hold.

The gain of regularity will be obtained by writing the equations in a specific gauge. Like for the conformal dimension it happens that the gauges in which we will get optimal regularity for the Stationary Yang-Mills Fields are Coulomb gauges (in such a gauge the equation (1.1) in $A$ becomes elliptic). In [14] a Coulomb gauge extraction theorem is given for curvatures in $L^{n / 2}$ and $D$ in $\mathfrak{U}_{1}^{n / 2}$. This assumption is stronger compare to the assumption on the connection of being stationary Yang-Mills in $\mathfrak{M}_{2}^{1,2} \cap \mathfrak{M}_{1}^{0,4}$ satisfying the approximability property (1.7). We have here the following result.

Theorem 1.3 Let $M=B^{n}, G \subset S O(l)$ and $n \geq 4$. There exists $\kappa(n)>0$, such that, for any 1-form $\tilde{A}$ in $M_{2}^{1,2}\left(B_{1}, \mathfrak{G}\right) \cap M_{1}^{0,4}\left(B_{1}, \mathfrak{G}\right)$, that can be approximated in $W^{1,2} \cap L^{4}$ by a sequence of smooth forms $\tilde{A}_{i}$ verifying

$$
\left\|F_{\tilde{A}_{i}}\right\|_{M_{2}^{0,2}\left(B_{1}, \mathfrak{G}\right)}^{2}=\sup _{x \in B_{1}, r>0} \frac{1}{r^{n-4}} \int_{B_{1} \cap B_{r}(x)}\left|F_{\tilde{A}_{i}}\right|^{2} \leq \kappa(n)
$$

there exists a change of gauge $s \in M_{2}^{2,2}\left(B_{1}, G\right) \cap M_{1}^{1,4}\left(B_{1}, G\right)$ such that $A=s^{-1} d s+s^{-1} \tilde{A} s$ verifies

$$
\begin{gathered}
d^{*} A=0 \quad \text { in } B_{1}, \\
\left\langle A ; \frac{\partial}{\partial r}\right\rangle=0 \quad \text { on } \partial B_{1}, \\
\|A\|_{M_{2}^{1,2}\left(B_{1}, \mathfrak{G}\right)}+\|A\|_{M_{1}^{0,4}\left(B_{1}, \mathfrak{G}\right)} \leq C(n)\left\|F_{A}\right\|_{M_{2}^{0,2}\left(B_{1}, \mathfrak{G}\right)} .
\end{gathered}
$$

The above result generalises the classic Uhlenbeck Coulomb Gauge extraction result in the following sense : Observe that for any $A \in W^{1, \frac{n}{2}}$ one has

$$
\|A\|_{M_{2}^{1,2}}+\|A\|_{M_{1}^{0,4}} \leq C\|A\|_{W^{1, \frac{n}{2}}}
$$

and that

$$
\sup _{x \in B_{1}, r>0} \frac{1}{r^{n-4}} \int_{B_{1} \cap B_{r}(x)}\left|F_{A}\right|^{2} \leq C\left[\sup _{x \in B_{1}, r>0} \int_{B_{1} \cap B_{r}(x)}\left|F_{A}\right|^{n / 2}\right]^{4 / n} .
$$

The structure of the proof of Theorem 1.3 follows Uhlenbeck approach in [14] but it requires the introduction of a new ingredient that can be sketched this way : the control of the supremum among the Yang-Mills energy density

$$
1 / r^{n-4} \int_{B^{r}}\left|F_{d+A}\right|^{2}
$$


is converted into a control of the BMO norm of a closed 2-form $\xi$ such that $d^{*} \xi$ gives the Coulomb Gauge $A=d^{*} \xi$ and the $L^{4}$-norm control of $A$ is obtained through the following interpolation inequality. Let $\Omega$ be a regular bounded domain of $\mathbb{R}^{n}$ we denote by $W^{2,2}(\Omega)$ the space of maps in $L^{2}(\Omega)$ whose first and second derivatives are also in $L^{2}(\Omega)$ and $\operatorname{BMO}(\Omega)$ denotes the space of maps on $\Omega$ admitting an extension $\tilde{u}$ on the whole $\mathbb{R}^{n}$ such that

$$
\sup _{x \in \mathbb{R}^{n}, r>0} \frac{1}{\left|B_{r}^{n}\right|} \int_{B_{r}^{n}(x)}\left|\tilde{u}-\frac{1}{\left|B_{r}^{n}\right|} \int_{B_{r}^{n}(x)} \tilde{u}\right|=\|\tilde{u}\|_{B M O\left(\mathbb{R}^{n}\right)}<+\infty
$$

in that case we denote $\|u\|_{B M O(\Omega)}$ the infimum of $\|\tilde{u}\|_{B M O\left(\mathbb{R}^{n}\right)}$ among all possible extensions of $u$ to the whole $\mathbb{R}^{n}$. Under the previous notations we have for any dimension $n \geq 1$ :

Theorem 1.4 Let $\Omega$ be a regular bounded domain of $\mathbb{R}^{n}$, there exists a constant $C(\Omega)$, such that, for any $u$ in $B M O \cap W^{2,2}(\Omega)$ we have

$$
\|\nabla u\|_{L^{4}(\Omega)}^{2} \leq C(\Omega)\|u\|_{B M O(\Omega)}\|u\|_{W^{2,2}(\Omega)}
$$

In the case where the BMO norm of $u$ is replaced by it's $L^{\infty}$ norm, this interpolation inequality is a standard Gagliardo-Nirenberg type inequality for Sobolev norms (see [3] for instance). It happens that the inequality above can even be improved by replacing in (1.11) the BMO norm of $u$ by the norm of $\nabla u$ in the Besov maximal Space $B_{\infty}^{-1, \infty}$ that contains derivatives of BMO function and inequality (1.11) enters in the family of improved Sobolev inequalities introduced in [6] (see section 2 below).

\section{Proof of Theorem 1.4}

We first recall the definition of the "maximal space" the homogeneous Besov space $\dot{B}_{\infty}^{-1, \infty}$ which is made of tempered distributions $f$ in $\mathcal{S}^{\prime}\left(\mathbb{R}^{n}\right)$ verifying

$$
\sup _{\left\{a>0 ; b \in \mathbb{R}^{n}\right\}} a\left|\left\langle f, g_{a, b}\right\rangle\right| \leq C<+\infty
$$

where $g$ is any given function in $\mathcal{S}$ normalized such that $\int_{\mathbb{R}^{n}} g=1$ (take for instance $\left.g(x)=(2 \pi)^{-n / 2} \exp \left(-|x|^{2} / 2\right)\right)$ and $g_{(a, b)}(x)=\frac{1}{a^{n}} g\left(\frac{x-b}{a}\right)$. It is a maximal space among the functional spaces whose norms are invariant under translation verifying also $\|f(\lambda x)\|=\lambda^{-1}\|f\|$. For instance, for $n \geq 2$, $f(x)=|x|^{-1} \in \dot{B}_{\infty}^{-1, \infty}$, or if $f(x)=\partial_{1} b_{1}(x)+\cdots+\partial_{n} b_{n}(x)$ where $b_{1}, \ldots, b_{n} \in$ $\operatorname{BMO}\left(\mathbb{R}^{n}\right)$ then $f \in \dot{B}_{\infty}^{-1, \infty}$. 
Definition 2.3 Let $\Omega$ be an arbitrary open subset of $\mathbb{R}^{n}$, then $C^{-1}(\Omega)$ is the space of restrictions to $\Omega$ of functions $f$ in $\dot{B}_{\infty}^{-1, \infty}$. The norm of $f \in C^{-1}(\Omega)$ is the infimum among the norm in $\dot{B}_{\infty}^{-1, \infty}\left(\mathbb{R}^{n}\right)$ of extensions $\tilde{f}$ of $f$ to the whole of $\mathbb{R}^{n}$ into a function of $\dot{B}_{\infty}^{-1, \infty}\left(\mathbb{R}^{n}\right)$.

This part is devoted to the proof of the following result

Theorem 2.5 Let $\Omega \subset \mathbb{R}^{n}$ be a connex bounded open and regular subset of $\mathbb{R}^{n}$. There exists a constant $C(\Omega)$ such that, for any function $f$ belonging to both $H^{1}(\Omega)$ and $C^{-1}(\Omega)$, we have

$$
\left\|f-m_{\Omega} f\right\|_{L^{4}(\Omega)}^{2} \leq C(\Omega)\|\nabla f\|_{L^{2}(\Omega)}\|f\|_{C^{-1}(\Omega)}
$$

where

$$
m_{\Omega} f=\frac{1}{|\Omega|} \int_{\Omega} f(x) d x
$$

is the average of $f$ on $\Omega$.

$H^{1}(\Omega)$ denotes here the Sobolev space of functions $f$ in $L^{2}(\Omega)$ such that $\nabla f \in L^{2}(\Omega)$.

Remark 2.1 One verifies easily the the constant $C(\Omega)$ above is invariant under translations and dilations.

Before proving Theorem 2.5 on arbitrary domain $\Omega$ we give the proof of the corresponding identity on the whole $\mathbb{R}^{n}$.

Precisely we prove

Theorem 2.6 Let $f$ be in $H^{1}\left(\mathbb{R}^{n}\right) \cap \dot{B}_{\infty}^{-1, \infty}\left(\mathbb{R}^{n}\right)$, then $f$ is in $L^{4}\left(\mathbb{R}^{n}\right)$ and we have

$$
\|f\|_{L^{4}\left(\mathbb{R}^{n}\right)}^{2} \leq C\|f\|_{H^{1}\left(\mathbb{R}^{n}\right)}\|f\|_{\dot{B}_{\infty}^{-1, \infty}\left(\mathbb{R}^{n}\right)} .
$$

Proof (of Theorem 2.6). We use the Littlewood-Paley decomposition of $f$ and write $f=\sum_{-\infty}^{+\infty} \Delta_{j}(f)$. We have (Littlewood-Paley)

$$
\begin{aligned}
\|f\|_{4} & \simeq\left\|\left(\sum\left|\Delta_{j}(f)\right|^{2}\right)^{\frac{1}{2}}\right\|_{4}=\left(\int_{\mathbb{R}^{n}}\left(\sum\left|\Delta_{j}(f)\right|^{2}\right)^{2} d x\right)^{1 / 4} \\
& \leq\left(\sum_{j} \sum_{j^{\prime} \geq j} \int_{\mathbb{R}^{n}}\left|\Delta_{j}(f)\right|^{2}\left|\Delta_{j^{\prime}}(f)\right|^{2} d x\right)^{1 / 4}
\end{aligned}
$$

Using the fact that $f \in \dot{B}_{\infty}^{-1, \infty}\left(\mathbb{R}^{n}\right)$, we have that

$$
\sup _{j} 2^{-j}\left\|\Delta_{j}(f)\right\|_{\infty}=\|f\|_{\dot{B}_{\infty}^{-1, \infty}\left(\mathbb{R}^{n}\right)}<+\infty .
$$


From (2.14) we then deduce

$$
\begin{aligned}
\|f\|_{4} & \leq C\|f\|_{\dot{B}_{\infty}^{-1, \infty}}^{1 / 2}\left(\sum_{j} \sum_{j^{\prime} \geq j} \int_{\mathbb{R}^{n}} 4^{j}\left|\Delta_{j^{\prime}}(f)\right|^{2}\right)^{1 / 4} \\
& \leq C\|f\|_{\dot{B}_{\infty}^{-1, \infty}}^{1 / 2}\left(\sum_{j^{\prime}} 4^{j^{\prime}}\left\|\Delta_{j^{\prime}}(f)\right\|_{2}^{2}\right)^{1 / 4} \leq C\|f\|_{\dot{B}_{\infty}^{-1, \infty}}^{1 / 2}\|f\|_{H^{1}\left(\mathbb{R}^{n}\right)}^{1 / 2}
\end{aligned}
$$

This ends the proof of Theorem 2.6.

Proof (of Theorem 2.5). We first handle the case of the half plane $\mathbb{R}_{+}^{n}=$ $\left\{\left(x_{1}, \ldots, x_{n}\right)\right.$ s.t. $\left.x_{n} \geq 0\right\}$. We first pave the half space by Whitney cubes

$$
\prod_{j=1}^{n-1}\left[p_{j} 2^{-i},\left(p_{j}+1\right) 2^{-i}\right] \times\left[2^{-j}, 2^{-j+1}\right] .
$$

Call $Q$ these cubes, $x_{Q}$ their center and $d_{Q}=2^{-i}$ their size. We have $1=\sum_{Q} \phi_{Q}$ on $\mathbb{R}_{+}^{n}$ where

$$
\phi_{Q}(x)=\phi\left(\frac{x-x_{Q}}{d_{Q}}\right)
$$

and $\phi$ is a compactly supported function in the unit reference cube $\tilde{Q}_{0}=$ $[-2,2]^{n}$ equal to one on the cube of half size $\frac{1}{2} \tilde{Q}_{0}=[-1,1]^{n}$. Consider also

$$
\theta_{Q}=d_{Q}^{-n} \theta\left(\frac{x-x_{Q}}{d_{Q}}\right)
$$

for a given function $\theta \in C_{0}^{\infty}\left(\frac{1}{2} \tilde{Q}_{0}\right)$ verifying $\int \theta=1$.

The proof of the theorem rely on several lemmas. First of all we have the following elementary lemma.

Lemma 2.1 The Banach space $\dot{B}_{\infty}^{-1, \infty}\left(\mathbb{R}^{n}\right)$ is a module over the Schwartz space $\mathcal{S}\left(\mathbb{R}^{n}\right)$ for $n \geq 1$. More precisely if we denote $\|\cdot\|_{*}$ the norm on $\dot{B}_{\infty}^{-1, \infty}\left(\mathbb{R}^{n}\right)$, for any $g$ in $\mathcal{S}\left(\mathbb{R}^{n}\right)$ there exists $C_{g}$ such that for any $R>0$ the following holds

$$
\left\|g\left(\frac{x}{R}\right) f(x)\right\|_{*} \leq C\|f\|_{*} .
$$

Consider now a function $f \in \dot{H}^{1}\left(\mathbb{R}_{+}^{n}\right)$. Write $f=u+v$ where

$$
\left\{\begin{array}{l}
u=\sum_{Q} \gamma_{Q} \phi_{Q} \quad \text { where } \gamma_{Q}=\int f \theta_{Q} d x \\
v=\sum_{Q}\left(f-\gamma_{Q}\right) \phi_{Q}
\end{array}\right.
$$


We will handle $u$ and $v$ independently. First of all we have :

Proposition 2.1 Under the notations above we have

$$
\|u\|_{\dot{H}^{1}} \leq C\|f\|_{\dot{H}^{1}}
$$

and

$$
\sum_{Q} \int_{Q}\left|f-\gamma_{Q}\right|^{2}\left|\nabla \phi_{Q}\right|^{2} d x \leq C\|\nabla f\|_{2}^{2}
$$

Proof (of Proposition 2.1). Poincaré inequality for $f$ reads

$$
\left(\int_{Q}\left|f-\gamma_{Q}\right|^{p} d x\right)^{1 / p} \leq C\left(\int_{Q}|\nabla f|^{2} d x\right)^{1 / 2}
$$

where $1 / 2-1 / p=1 / n$ and $\gamma_{Q}=\int f \theta_{Q} d x$. In fact the usual Poincaré inequality involves the standard average $m_{Q}$ of $f$ on $Q$, but observe that

$$
\left(\int_{Q}\left|\gamma_{Q}-m_{Q}\right|^{p} d x\right)^{1 / p}=\left|\gamma_{Q}-m_{Q}\right||Q|^{1 / p}
$$

and

$$
\left|\gamma_{Q}-m_{Q}\right| \leq \frac{1}{|Q|} \int_{Q}\left|f(x)-m_{Q}\right| d x \leq\left(\frac{1}{|Q|} \int_{Q}\left|f-m_{Q}\right|^{p} d x\right)^{1 / p} .
$$

From Poincaré (2.20) we deduce

$$
\int_{Q}\left|f-\gamma_{Q}\right|^{2} d x \leq C d_{Q}^{2} \int_{Q}|\nabla f|^{2} d x
$$

and the proposition follows.

As a result of proposition 2.1 we have that $v \in H^{1}\left(\mathbb{R}_{+}^{n}\right)$ and it follows that $u \in H^{1}\left(\mathbb{R}_{+}^{n}\right)$ also.

Apply now Theorem 2.6 to $\left(f-\gamma_{Q}\right) \phi_{Q}=F_{Q}$. We have

$$
\left\|F_{Q}\right\|_{4}^{4} \leq C\left\|\nabla F_{Q}\right\|_{2}^{2}\left\|F_{Q}\right\|_{*}^{2}
$$

In one hand we have, using proposition $2.1, \sum_{Q}\left\|\nabla F_{Q}\right\|_{2}^{2} \leq C\|\nabla f\|_{2}^{2}$. In the other hand we write

$$
\left\|F_{Q}\right\|_{*} \leq\left\|f \phi_{Q}\right\|_{*}+\left|\gamma_{Q}\right|\left\|\phi_{Q}\right\|_{*}
$$


and from the definition of $\gamma_{Q}=\int f \theta_{Q} d x$ we have $\left|\gamma_{Q}\right| \leq\|f\|_{*} d_{Q}^{-1}$. $\left\|f \phi_{Q}\right\|_{*}$ is estimated using Lemma 2.1 and we finally obtain

$$
\|v\|_{4}^{4} \leq C\|\nabla f\|_{2}^{2}\|f\|_{*}^{2}
$$

Let now establish the same identity but for $u$. We first observe that

$$
|u(x)| \leq \sum_{Q} \frac{\|f\|_{*}}{d_{Q}}\left|\phi_{Q}(x)\right| \leq C \frac{\|f\|_{*}}{x_{n}}
$$

Estimate (2.23) for $u$ will then follow from the following lemma and Theorem 2.5 will be proved in the case of the half space.

Lemma 2.2 Let $f$ be a function from $\mathbb{R}_{+}^{n}$ into $\mathbb{C}$ verifying

$$
|f(x)| \leq \frac{m}{x_{n}}
$$

and

$$
\left(\int_{\mathbb{R}_{+}^{n}}|\nabla f|^{2}\right)^{1 / 2} \leq M
$$

then we have

$$
\|f\|_{L^{4}} \leq \sqrt{m M}
$$

Proof (of Lemma 2.2). Let first study the one dimensionnal case and write $t=x_{n}$. We have

$$
\begin{aligned}
& \int_{0}^{\infty}|f|^{4} d t \leq \int_{0}^{\left(\frac{m}{M}\right)^{2 / 3}}|f|^{4} d t+\int_{\left(\frac{m}{M}\right)^{2 / 3}}^{\infty}|f|^{4} d t \\
& \quad \leq\left(\frac{m}{M}\right)^{2 / 3} \sup _{\left[0,\left(\frac{m}{M}\right)^{2 / 3}\right]}|f|^{4}+m^{4}\left(\frac{m}{M}\right)^{-2}
\end{aligned}
$$

From hypothesis (2.25) we have $\left|f\left(\left(\frac{m}{M}\right)^{2 / 3}\right)\right| \leq M^{2 / 3} m^{1 / 3}$ and

$$
\left|f(t)-f\left(t^{\prime}\right)\right| \leq M \sqrt{t^{\prime}-t} \leq\left(\frac{m}{M}\right)^{1 / 3} M=m^{1 / 3} M^{2 / 3} .
$$

Therefore $\|f\|_{\infty} \leq 2 M^{2 / 3} m^{1 / 3}$ and inserting this estimate in (2.28) we get the result for $n=1$. In arbitrary dimension we simply integrate in $x^{\prime}=\left(x_{1}, \ldots, x_{n-1}\right)$ the inequality established in 1 dimension and Lemma 2.2 is proved. 
Combining (2.24) and the lemma above we have established that

$$
\|u\|_{4}^{4} \leq C\|\nabla f\|_{2}^{2}\|f\|_{*}^{2}
$$

Combining now (2.23) and (2.29), Theorem 2.5 is now established on the half space.

Consider now a regular bounded domain of $\mathbb{R}^{n}$ and take $f \in H^{1}(\Omega) \cap$ $\dot{B}_{\infty}^{-1, \infty}(\Omega)$. We use an appropriate partition of unity of $\Omega$ and diffeomorphisms to reduce the problem to the case of the whole $\mathbb{R}^{n}$ or $\mathbb{R}_{+}^{n}$ and Theorem 2.5 is proved.

\section{Proof of Theorem 1.3}

We first prove Theorem 1.3 where the norms $M_{2}^{1,2}$ and $M_{1}^{0,4}$ are replaced by the norms $M_{2-\alpha}^{1,2}$ and $M_{1-\alpha}^{0,4}$ for some $\alpha>0$. Precisely we have

Theorem 3.7 Let $M=B^{n}, G \subset S O(l), n \geq 4$ and $\alpha>0$. There exist $\kappa(n)>0$ and $C(n)$, independent on $\alpha$, such that, for any 1-form $\tilde{A}$ in $M_{2-\alpha}^{1,2}\left(B_{1}, \mathfrak{G}\right) \cap M_{1-\alpha}^{0,4}\left(B_{1}, \mathfrak{G}\right)$, if we denote $F_{\tilde{A}}=d \tilde{A}+[\tilde{A}, \tilde{A}]$,

$$
\sup _{x \in B_{1}, r>0} \frac{1}{r^{n-4}} \int_{B_{1} \cap B_{r}(x)}\left|F_{\tilde{A}}\right|^{2} \leq \kappa(n)
$$

then there exists a change of gauge $s \in M_{2-\alpha}^{2,2}\left(B_{1}, G\right) \cap M_{1-\alpha}^{1,4}\left(B_{1}, G\right)$ such that $A=s^{-1} d s+s^{-1} \tilde{A} s$ verifies

$$
\begin{gathered}
d^{*} A=0 \quad \text { in } B_{1} \\
\left\langle A ; \frac{\partial}{\partial r}\right\rangle=0 \quad \text { on } \partial B_{1} \\
\|A\|_{M_{2}^{1,2}\left(B_{1}, \mathfrak{G}\right)}+\|A\|_{M_{1}^{0,4}\left(B_{1}, \mathfrak{G}\right)}^{2} \leq C(n)\left\|F_{A}\right\|_{M_{2}^{0,2}\left(B_{1}, \mathfrak{G}\right)}
\end{gathered}
$$

and

$$
\|A\|_{M_{2-\alpha}^{1,2}\left(B_{1}, \mathfrak{G}\right)}+\|A\|_{M_{1-\alpha}^{0,4}\left(B_{1}, \mathfrak{G}\right)}^{2} \leq C(n)\left\|F_{A}\right\|_{M_{2-\alpha}^{0,2}\left(B_{1}, \mathfrak{G}\right)}
$$

Proof (of Theorem 3.7). We follow the strategy adopted in [14] for proving Theorem 2.1. Consider

$$
\mathfrak{S}_{\kappa}^{\alpha}=\left\{\tilde{A} \in M_{2-\alpha}^{1,2}\left(B_{1}\right) \cap M_{1-\alpha}^{0,4}\left(B_{1}\right) \quad \text { s.t. } \sup _{x \in B_{1}, r>0} \frac{1}{r^{n-4}} \int_{B_{1} \cap B_{r}(x)}\left|F_{\tilde{A}}\right|^{2} \leq \kappa\right\}
$$

The goal is to prove that for $\kappa$ small enough, independent on $\alpha$, the set of $\tilde{A}$ in $\mathfrak{S}_{\kappa}^{\alpha}$ gauge equivalent to some $A$ verifying $(3.30), \ldots,(3.33)$ is both open 
and closed for some $C(n)$. Once this will be established it remains to prove that smooth connections in $\mathfrak{S}_{\kappa}^{\alpha}$ are in the connected component of any flat connection in $\mathfrak{S}_{\kappa}^{\alpha}$ to get the desired result. We start with the connectivity property.

Lemma $3.3 \mathfrak{S}_{\kappa}^{\alpha}$ is path connected for the $M_{2-\beta}^{1,2} \cap M_{1-\beta}^{0,4}$ topology for any $0<\beta<\alpha$.

Proof (of Lemma 3.3). As in Lemma 2.3 of [14] we use the following path $t \rightarrow D=D_{0}+t A(t x)$ for $t \in[0,1]$ and $D_{0}$ a flat reference connection. Clearly we have for any $x_{0} \in B_{1}$ and any $r>0$

$$
\frac{1}{r^{n-4}} \int_{B_{r}\left(x_{0}\right) \cap B_{1}}\left|F_{D_{t}}\right|^{2}=\frac{1}{(t r)^{n-4}} \int_{B_{t r}\left(t x_{0}\right) \cap B_{t}(0)}\left|F_{D}\right|^{2} \leq \kappa
$$

Let $t_{0} \in[0,1), \varepsilon>0$ and $r_{\varepsilon}>0$ such that $r_{\varepsilon}^{\alpha-\beta}=\varepsilon$ and let $A_{\delta}$ be a smooth approximation of $A$ that converges to $A$ in $L^{4} \cap W^{1,2}\left(B_{1}\right)$. We choose $\delta$ small enough so that

$$
\int_{B_{1}}\left|A_{\delta}(x)-A(x)\right|^{4}+\left|\nabla\left(A_{\delta}(x)-A(x)\right)\right|^{2} \leq r_{\varepsilon}^{n-4+\beta}
$$

and once $\delta$ is fixed we choose $\left|t-t_{0}\right|$ small enough so that $\| A_{\delta}(t x)-$ $A_{\delta}\left(t_{0} x\right)\left\|_{\infty}+\right\| \nabla\left(A_{\delta}(x)-A(x)\right) \|_{\delta} \leq \varepsilon$.

For $r>r_{\varepsilon}$ we have

$$
\begin{aligned}
\frac{1}{r^{n-4+\beta}} & \int_{B_{r}\left(x_{0}\right) \cap B_{1}}\left|t A(t x)-t_{0} A\left(t_{0} x\right)\right|^{4} \leq \frac{1}{r^{n-4+\beta}} \int_{B_{r}\left(x_{0}\right) \cap B_{1}}\left|t A(t x)-t A_{\delta}(t x)\right|^{4} \\
& +\frac{1}{r^{n-4+\beta}} \int_{B_{r}\left(x_{0}\right) \cap B_{1}}\left|t A_{\delta}(t x)-t_{0} A_{\delta}\left(t_{0} x\right)\right|^{4}+\left|t_{0} A_{\delta}\left(t_{0} x\right)-t_{0} A\left(t_{0} x\right)\right|^{4} \\
& \leq C \varepsilon
\end{aligned}
$$

and for $r<r_{\varepsilon}$ we have

$$
\frac{1}{r^{n-4+\beta}} \int_{B_{r}\left(x_{0}\right) \cap B_{1}}\left|t A(t x)-t_{0} A\left(t_{0} x\right)\right|^{4} \leq r^{\alpha-\beta}\|A\|_{M_{1-\alpha}^{0,4}} \leq C \varepsilon .
$$

This proves that the path $t \rightarrow D_{t}$ connect continuously in $\mathfrak{M}_{1-\beta}^{0,4} \cap \mathfrak{M}_{2-\beta}^{1,2}$ $D \in \mathfrak{M}_{1-\alpha}^{0,4} \cap \mathfrak{M}_{2-\alpha}^{1,2}$ and $D_{0}$ and Lemma 3.3 is proved. 


\subsection{The closedness property}

This subsection is devoted to the proof of the following result :

Lemma 3.4 For $\alpha \geq 0$, the set of $\tilde{A}$ in $\mathfrak{S}_{\kappa}^{\alpha}$, gauge equivalent to some $A$ satisfying (3.30), ..., (3.33), is closed for $\kappa(n)$ small enough and $C(n)$ large enough.

As in the proof of Lemma 2.4 of [14] it is quite straightforward to prove that for $\tilde{A}$, to be gauge equivalent to some $A$ satisfying (3.30) and (3.31), passes to the limit under strong convergence in $M_{2-\alpha}^{1,2} \cap M_{1-\alpha}^{0,4}$ for $\alpha \geq 0$. So we just need to prove the following

Lemma 3.5 There exist $k(n)>0$ and $C(n)>0$ such that if $A$ satisfies $d^{*} A=0$ on $B_{1}, \iota_{\partial B_{1}}^{*} * A=0$ ( $\iota_{\partial B_{1}}$ is the embedding of $\partial B_{1}$ in $\left.\mathbb{R}^{n}\right)$ and

$$
\sup _{x \in B_{1}, r>0} \frac{1}{r^{n-4}} \int_{B_{r}(x) \cap B_{1}}|A|^{4} \leq k(n)
$$

then for any $\alpha \geq 0$ we have

$$
\|A\|_{M_{2-\alpha}^{1,2}\left(B_{1}, \mathfrak{G}\right)}+\|A\|_{M_{1-\alpha}^{0,4}\left(B_{1}, \mathfrak{G}\right)} \leq C(n)\left\|F_{A}\right\|_{M_{2-\alpha}^{0,2}\left(B_{1}, \mathfrak{G}\right)}
$$

Proof (of Lemma 3.5). There exists a unique $\xi$ solving

$$
\begin{cases}A=d^{*} \xi & \text { in } B_{1} \\ d \xi=0 & \text { in } B_{1} \\ \iota_{\partial B_{1}}^{*} * \xi=0 & \end{cases}
$$

( $\xi$ minimizes $\int_{B_{1}}\left|A-d^{*} \xi\right|^{2}$ among the $\xi$ solving $d \xi=0$ and $\iota_{\partial B_{1}}^{*} * \xi=0$ ). $\xi$ solves in particular

$$
\Delta \xi+\left[d^{*} \xi, d^{*} \xi\right]=F_{A}
$$

Let $\phi(x)=x /|x|^{2}$ from $B_{1} \backslash B_{1 / 2}$ into $B_{2} \backslash B_{1}$. Denote by $g$ the metric, pulled back by $\phi^{-1}$ of the standard metric $g_{0}=\delta_{i, j} d x_{i} \otimes d x_{j}$ in $B_{1} \backslash B_{1 / 2}\left(g_{i, j}=\frac{\delta_{i, j}}{|x|^{4}}\right)$ so that $\phi$ becomes a negative isometry from $B_{1} \backslash B_{1 / 2}$ into $B_{2} \backslash B_{1}$. Denote also by $\hat{g}$ the metric equal to $g_{0}$ on $B_{1}$ and $g$ in $B_{2} \backslash B_{1}$. Let $\hat{\xi}$ defined by

$$
\begin{cases}\hat{\xi}=\xi & \text { in } B_{1}, \\ \hat{\xi}=\phi^{*} \xi & \text { in } B_{1} .\end{cases}
$$

Since $\iota_{\partial B_{1}}^{*} * \xi=0$ on $\partial B_{1}$ we have $<\xi ; \frac{\partial}{\partial r}>=0$ on $\partial B_{1}$, thus $\hat{\xi}=\phi^{*} \xi=\xi$ on $\partial B_{1}$. Therefore it is easy to deduce that

$$
d \hat{\xi}=0 \quad \text { in } B_{2} .
$$


Since $\phi$ realizes an isometry we have $\phi^{*} d^{*_{0}} \xi=d^{* g} \hat{\xi}$ on $B_{2} \backslash B_{1}$. Moreover, since $\iota_{\partial B_{1}}^{*} * \xi=0, \iota_{\partial B_{1}}^{*} * d^{*} \xi=\iota_{\partial B_{1}}^{*} d * \xi=0$ where $*$ means $*_{g_{0}}$. Therefore $d^{*} \xi$ on $\partial B_{1}$ has no component along $d r$ and this implies that $\phi^{*} d^{*} \xi=d^{*} \xi$ on $\partial B_{1}$. So we deduce that for any $\psi \in C_{0}^{\infty}\left(\wedge^{n-2} B_{2}\right)$ we have

$$
\int_{B_{2}} d^{* g} \hat{\xi} \wedge d \psi=\int_{B_{2} \backslash B_{1}} d \phi^{*} d^{* g_{0}} \xi \wedge \psi+\int_{B_{1}} d d^{*_{0}} \xi \wedge \psi
$$

Combining (3.38) and (3.39) we get

$$
\Delta_{\hat{g}} \hat{\xi}+\left[d^{*} \hat{g} \hat{\xi}, d^{*} \hat{g} \hat{\xi}\right]=\hat{F}_{A}
$$

where $\hat{F}_{A}=F_{A}$ in $B_{1}$ and $\hat{F}_{A}=\phi^{*} F_{A}$ in $B_{2} \backslash B_{1}$. We will denote also $\hat{A}=A$ in $B_{1}$ and $\hat{A}=\phi^{*} A$ in $B_{2} \backslash B_{1}$ so $\hat{A}=d^{*} \hat{\xi} \hat{\xi}$. Clearly

$$
\left\|\hat{F}_{A}\right\|_{M_{2-\alpha}^{0,2}\left(B_{2}\right)} \leq C\left\|F_{A}\right\|_{M_{2-\alpha}^{0,2}\left(B_{1}\right)} \text { and }\|\hat{A}\|_{M_{1-\alpha}^{0,4}\left(B_{2}\right)} \leq C\|A\|_{M_{1-\alpha}^{0,4}\left(B_{1}\right)}
$$

Therefore, combining (3.40) and (3.41), we have

$$
\begin{aligned}
\left\|\Delta_{\hat{g}} \hat{\xi}\right\|_{M_{2-\alpha}^{0,2}\left(B_{2}\right)} & \leq C\left\|F_{A}\right\|_{M_{2-\alpha}^{0,2}\left(B_{1}\right)}+C\|A\|_{M_{1}^{0,4}\left(B_{1}\right)}\left\|d^{*} \xi\right\|_{M_{1-\alpha}^{0,4}\left(B_{1}\right)} \\
& \leq C\left\|F_{A}\right\|_{M_{2-\alpha}^{0,2}\left(B_{1}\right)}+C k(n)\left\|d^{*} \xi\right\|_{M_{1-\alpha}^{0,4}\left(B_{1}\right)}
\end{aligned}
$$

We shall use the following lemma.

Lemma 3.6 Let $\beta \in[0,1)$, let $g$ be an arbitrary smooth metric on $B_{2}$ and $u$ a map on $B_{2}$, there exists $C_{g}>0$, independent on $u$, such that

$$
\begin{aligned}
& \sup _{B_{r}(x) \subset B_{1}} \frac{1}{r^{n-2+\beta}} \int_{B_{r}(x)}|\nabla u|_{g}^{2} d v o l_{g} \leq \\
& \quad \leq C_{g} \sup _{B_{r}(x) \subset B_{2}} \frac{1}{r^{n-4+\beta}} \int_{B_{r}(x)}\left|\Delta_{g} u\right|^{2} d v o l_{g} \quad+C_{g} \int_{B_{2}}|\nabla u|_{g}^{2} d v o l_{g}
\end{aligned}
$$

Proof (of Lemma 3.6). Let $B_{r}(x) \subset B_{2}$ and $\rho \leq r$. We consider $v$ the solution of

$$
\begin{cases}\Delta_{g} v=0 & \text { in } B_{r}(x) \\ v=u & \text { on } \partial B_{r}(x)\end{cases}
$$

Standard elliptic estimates on harmonic functions imply

$$
\forall \rho \leq r \quad \int_{B_{\rho}(x)}|\nabla v|^{2} \leq C\left(\frac{\rho}{r}\right)^{n} \int_{B_{r}(x)}|\nabla v|^{2}
$$


Let $w=u-v . w$ solves

$$
\begin{cases}\Delta_{g} w=\Delta_{g} u & \text { in } B_{r}(x) \\ w=0 & \text { on } \partial B_{r}(x)\end{cases}
$$

Multiplying (3.45) by $w$ and integrating by parts over $B_{r}(x)$ gives

$$
\begin{aligned}
\int_{B_{r}(x)}|\nabla w|^{2} & \leq C\left(\int_{B_{r}(x)}|w|^{2}\right)^{1 / 2}\left(\int_{B_{r}(x)}\left|\Delta_{g} w\right|^{2}\right)^{1 / 2} \\
& \leq C r^{2} \int_{B_{r}(x)}\left|\Delta_{g} w\right|^{2}
\end{aligned}
$$

(3.44), (3.45) and (3.46) imply

$$
\int_{B_{\rho}(x)}|\nabla u|^{2} \leq C\left(\frac{\rho}{r}\right)^{n} \int_{B_{r}(x)}|\nabla u|^{2}+C r^{2} \int_{B_{r}(x)}\left|\Delta_{g} u\right|^{2}
$$

Let $T(\rho)=\frac{1}{\rho^{n-2+\alpha}} \int_{B_{\rho}(x)}|\nabla u|^{2}$. (3.47) becomes

$$
T(\rho) \leq C\left(\frac{\rho}{r}\right)^{2-\alpha} T(r)+C\left(\frac{r}{\rho}\right)^{n-2+\alpha}\left\|\Delta_{g} u\right\|_{M_{2-\alpha}^{0,2}\left(B_{1}\right)}^{2}
$$

Let $\lambda$ be a number between 0 and 1 depending only on $\alpha$ such that $C \lambda^{2-\alpha} \leq \frac{1}{2}$, we have then for any $B_{r}(x) \subset B_{2}$

$$
T(\lambda r) \leq \frac{1}{2} T(r)+C\left\|\Delta_{g} u\right\|_{M_{2-\alpha}^{0,2}\left(B_{1}\right)}^{2}
$$

Let $i=\left[\frac{\log r}{\log \lambda}\right]$, the result is obtained by iterating the above identity between $r=1$ and $r=\lambda^{i}$.

\section{End of the proof of Lemma 3.5 :}

From Lemma 3.6 and (3.42) we deduce that

$$
\sup _{B_{r}(x) \subset B_{3 / 2}} \frac{1}{r^{n-2+\alpha}} \int_{B_{r}(x)}|\nabla \hat{\xi}|^{2} \leq
$$

$$
\leq C\left\|F_{A}\right\|_{M_{2-\alpha}^{0,2}\left(B_{1}\right)}^{2}+C k(n)\left\|d^{*} \xi\right\|_{M_{1-\alpha}^{0,4}\left(B_{1}\right)}^{2}+\int_{B_{1}}|\nabla \xi|^{2}
$$

From (3.36), by the mean of standard elliptic estimates we have

$$
\int_{B_{1}}|\xi|^{2}+\int_{B_{1}}|\nabla \xi|^{2} \leq C\left\|F_{A}\right\|_{L^{2}\left(B_{1}\right)}^{2}+C k(n)\|\nabla \xi\|_{L^{4}\left(B_{1}\right)}^{2}
$$


We handle now the case $\alpha>0$. From Theorem 1.2, Chapter III of [7], we have for any ball $B_{r}(y) \subset B_{5 / 4}$

$$
\begin{gathered}
\|\hat{\xi}-\hat{\xi}(y)\|_{L^{\infty}\left(B_{r}(y)\right)}^{2} \leq C r^{2 \alpha} \sup _{B_{r}(x) \subset B_{\frac{3}{2}}} \frac{1}{r^{n-2+2 \alpha}} \int_{B_{r}(x)}|\nabla \hat{\xi}|^{2} \\
\leq C r^{2 \alpha}\left[\left\|F_{A}\right\|_{M_{2-\alpha}^{0,2}\left(B_{1}\right)}^{2}+k(n)\|\nabla \xi\|_{M_{1-\alpha}^{0,4}\left(B_{1}\right)}^{2}\right]
\end{gathered}
$$

Using now a Gagliardo-Nirenberg interpolation inequality we get for any $B_{r}(y) \subset B_{5 / 4}$

$$
\begin{aligned}
\int_{B_{r}(y)}|\nabla \hat{\xi}|^{4} \leq & C\|\hat{\xi}-\hat{\xi}(y)\|_{L^{\infty}\left(B_{r}(y)\right)}^{2} \int_{B_{r}(y)}|\Delta \hat{\xi}|^{2} \\
& +C r^{n-4}\|\hat{\xi}-\hat{\xi}(y)\|_{L^{\infty}\left(B_{r}(y)\right)}^{4}
\end{aligned}
$$

From this identity, (3.42), (3.50) and $k(n)$ small enough we obtain

$$
\|\nabla \xi\|_{M_{1-\alpha}^{0,4}\left(B_{1}\right)}^{4} \leq C\left\|F_{A}\right\|_{M_{2-\alpha}^{1,2}\left(B_{1}\right)}^{4}
$$

and we deduce (3.35) for $\alpha>0$.

The case $\alpha=0$. This is the sharp case where we make use of the interpolation inequality (1.11). Let $\overline{\Delta_{\hat{g}} \hat{\xi}}$ be the function equal to $\Delta_{\hat{g}} \hat{\xi}$ on $B_{3 / 2}$ and equal to zero elsewhere. denote $\bar{\xi}=\Delta_{\hat{g}}^{-1}\left(\overline{\Delta_{\hat{g}} \hat{\xi}}\right)$ where $\hat{g}$ is extended out of $B_{2}$ by interpolating $\frac{1}{|x|^{4}} \delta_{i, j}$ and $\delta_{i, j}$ between $B_{2}$ and $B_{4}$, moreover $\Delta_{\hat{g}}^{-1}$ is the convolution with the Green Kernel of $\Delta_{\hat{g}}$ on $\mathbb{R}^{n}$. It is clear that we have for instance

$$
\int_{B_{4}}|\bar{\xi}|^{2} \leq C \int_{B_{\frac{3}{2}}}\left|\Delta_{\hat{g}} \hat{\xi}\right|^{2}
$$

Finally we denote $\tilde{\xi}=\psi \bar{\xi}$ where $\psi$ is a smooth function equal to 1 in $B_{2}$ and equal to 0 in $\mathbb{R}^{n} \backslash B_{3}$. For instance we have the fact that the support of $\nabla \phi$ is contained in $B_{3} \backslash B_{2}$ where $\bar{\xi}$ is harmonic (for $\hat{g}$ ). Therefore, using (3.53), it is not difficult to check that

$$
\sup _{B_{r}(x) \subset \mathbb{R}^{n}} \frac{1}{r^{n-4}} \int_{B_{r}(x)}\left|\Delta_{\hat{g}} \tilde{\xi}\right|^{2} \leq C \sup _{B_{r}(x) \subset B_{2}} \frac{1}{r^{n-4}} \int_{B_{r}(x)}\left|\Delta_{\hat{g}} \hat{\xi}\right|^{2}
$$

Using now Lemma 3.6 we get that

$$
\sup _{B_{r}(x) \subset \mathbb{R}^{n}} \frac{1}{r^{n-2}} \int_{B_{r}(x)}|\nabla \tilde{\xi}|^{2} \leq C \sup _{B_{r}(x) \subset B_{2}} \frac{1}{r^{n-4}} \int_{B_{r}(x)}\left|\Delta_{\hat{g}} \hat{\xi}\right|^{2}
$$


Using Poincaré inequality we obtain from (3.55) and (3.40) that

$$
\begin{aligned}
\|\tilde{\xi}\|_{B M O\left(\mathbb{R}^{n}\right)}^{2} & \leq C \sup _{B_{r}(x) \subset B_{2}} \frac{1}{r^{n-4}} \int_{B_{r}(x)}\left|\Delta_{\hat{g}} \hat{\xi}\right|^{2} \\
& \leq C\left\|F_{A}\right\|_{M_{2}^{0,2}\left(B_{1}\right)}^{2}+C k(n)\left\|d^{*} \xi\right\|_{M_{1}^{0,4}\left(B_{1}\right)}^{2}
\end{aligned}
$$

Using now inequality (1.11) on a ball $B_{r}(x)$ in $\mathbb{R}^{n}$ we have

$$
\int_{B_{r}(x)}|\nabla \tilde{\xi}|^{4} \leq C\|\tilde{\xi}\|_{B M O\left(\mathbb{R}^{n}\right)}^{2} \int_{B_{r}(x)}\left|\Delta_{g} \tilde{\xi}\right|^{2}+C r^{n-4}\|\tilde{\xi}\|_{B M O\left(\mathbb{R}^{n}\right)}^{4}
$$

Combining (3.56) and (3.57) we obtain

$$
\sup _{B_{r}(x) \subset \mathbb{R}^{n}} \frac{1}{r^{n-4}} \int_{B_{r}(x)}|\nabla \tilde{\xi}|^{4} \leq C\left\|F_{A}\right\|_{M_{2}^{0,2}\left(B_{1}\right)}^{4}+C k(n)\left\|d^{*} \xi\right\|_{M_{1}^{0,4}\left(B_{1}\right)}^{4}
$$

Using (3.49) and (3.58) we have

$$
\int_{B_{\frac{3}{2}}}|\nabla(\tilde{\xi}-\hat{\xi})|^{2} \leq C\left\|F_{A}\right\|_{M_{2-\alpha}^{0,2}\left(B_{1}\right)}^{2}+C k(n)\left\|d^{*} \xi\right\|_{M_{1-\alpha}^{0,4}\left(B_{1}\right)}^{2}
$$

and since $\tilde{\xi}-\hat{\xi}$ is harmonic on $B_{\frac{3}{2}}$, we obtain that

$$
\sup _{B_{r}(x) \subset B_{\frac{5}{4}}} \frac{1}{r^{n-4}} \int_{B_{r}(x)}|\nabla(\tilde{\xi}-\hat{\xi})|^{4} \leq C\left\|F_{A}\right\|_{M_{2}^{0,2}\left(B_{1}\right)}^{4}+C k(n)\left\|d^{*} \xi\right\|_{M_{1}^{0,4}\left(B_{1}\right)}^{4}
$$

Combining now (3.58) and (3.59) we obtain that

$$
\|\nabla \xi\|_{M_{1}^{0,4}\left(B_{1}\right)}^{4} \leq C\left\|F_{A}\right\|_{M_{2}^{0,2}\left(B_{1}\right)}^{4}
$$

and Lemma 3.5 is proved.

Remark 3.2 Observe that one could have use a slightly different strategy and developp a version of proposition 3.1 of [1] on a bounded domain as a substitute of the inequality (1.11).

\subsection{The openess property.}

This subsection is devoted to the proof of the following result

Lemma 3.7 For $\alpha>0$, the set of $\tilde{A}$ in $\mathfrak{S}_{\kappa}^{\alpha}$, gauge equivalent to some $A$ satisfying (3.30), ...,(3.33), is open for $\kappa(n)$ small enough and $C(n)$ large enough. 
First of all we will need the following lemma.

Lemma 3.8 There exists $k(n)>0$ such that for any $A \in \mathfrak{S}_{k(n)}^{0}$ verifying $d^{*} A=0$ and $\iota_{\partial B_{1}}^{*} A=0$ and any $\alpha>0$, there exists $\varepsilon>0$ such that for any one-form $\lambda \in \mathfrak{S}^{\alpha}$ verifying

$$
\|\lambda\|_{\mathfrak{S}^{\alpha}} \leq \varepsilon \quad \text { and } \quad \iota_{\partial B_{1}}^{*} \lambda=0
$$

then there exists a unique s from $B_{1}$ into $G$ such that

$$
\begin{aligned}
& d^{*}\left(s^{-1} d s+s^{-1}(A+\lambda) s\right)=0 \\
& \iota_{\partial B_{1}}^{*} d s=0 \\
& \left\|s^{-1} d s\right\|_{\mathfrak{S}^{\alpha}} \leq C\|\lambda\|_{\mathfrak{S}^{\alpha}}
\end{aligned}
$$

Proof (of Lemma 3.8). The proof is identical to the proof of Lemma 2.7 of [14] replacing the $W^{2, p}$ norm for $s(p>n / 2)$ by the norm $\mathfrak{S}^{\alpha}$ for $s^{-1} d s$. The main ingredient to transpose Uhlenbeck's proof to the present situation is to observe that, having derivatives in $\mathfrak{S}^{\alpha}$, implies that one is $C^{0}$ (for $\alpha>0$ ) (see [7]) and in particular this implies that the operator $s \rightarrow s^{-1}$ is smooth in this space. Therefore

$$
(U, \lambda) \longrightarrow d^{*}\left(s^{-1} d s+s^{-1}(A+\lambda) s\right)
$$

is smooth from the space of $s$ having derivatives in $\mathfrak{S}^{\alpha}$ into $M_{2-\alpha}^{0,2}\left(B_{1}\right)$ and the Linearization argument of [14] Lemma 2.7 may be applied.

Finally in order to deduce Lemma 3.7 from Lemma 3.8 we need the equivalent lemma to Lemma 2.6 in [14] in order to ensure the boundary condition. It is not difficult to see that this Lemma 2.6 extends naturally to the present setting where Sobolev spaces are replaced by Morrey spaces.

\subsection{Theorem 3.7 implies Theorem 1.3}

We will need the following consequence of Theorem 1.3 :

Lemma 3.9 Let $\kappa(n)$ given by Theorem 3.7 and let $\tilde{A}$ be a smooth 1-form solving

$$
\sup _{x \in B_{1}, r>0} \frac{1}{r^{n-4}} \int_{B_{1} \cap B_{r}(x)}\left|F_{\tilde{A}}\right|^{2} \leq \kappa(n)
$$

Consider the Coulomb gauge A given by Theorem 3.7 for some $\alpha>0$, it then solves, for any $\rho>0$,

$$
\sup _{x \in B_{1}, r>\rho} \frac{1}{r^{n-4}} \int_{B_{r}(x) \cap B_{1}}|A|^{4} \leq C(n) \sup _{x \in B_{1}, r>\rho} \frac{1}{r^{n-4}} \int_{B_{r}(x) \cap B_{1}}\left|F_{A}\right|^{2}
$$


Let $\xi$ solving (3.36). Like in the proof of Lemma 3.5 we extend $\xi$ by $\hat{x i}$, that we keep denoting $\xi$, this permits to forget the boundary of $B_{1}$. Let $x_{0} \in B_{1}$ and $\rho>0$. For any $r>\rho$ we consider $v$ and $w$ solving $\xi=v+w$ and

$$
\begin{cases}\Delta v=0 & \text { in } B_{r}\left(x_{0}\right) \\ v=\xi & \text { on } \partial B_{r}\left(x_{0}\right)\end{cases}
$$

Since $v$ is harmonic it solves

$$
\int_{B_{\rho}}|\nabla v|^{4} \leq C\left(\frac{\rho}{r}\right)^{n} \int_{B_{r}}|\nabla v|^{4}
$$

and $\|v\|_{B M O\left(B_{r}\left(x_{0}\right)\right)} \leq C\|\xi\|_{B M O\left(B_{1}\right)} \leq C k(n)$. Moreover since $w$ solves

$$
\begin{cases}\Delta w=\Delta \xi & \text { in } B_{r}\left(x_{0}\right) \\ w=0 & \text { on } \partial B_{r}\left(x_{0}\right)\end{cases}
$$

we have, by the mean of Lemma 3.6,

$$
\|w\|_{B M O\left(B_{r}\left(x_{0}\right)\right)}^{2} \leq C \sup _{x \in B_{r}\left(x_{0}\right), s<2 r} \frac{1}{s^{n-4}} \int_{B_{s}(x)}|\Delta \xi|^{2} \leq C k(n)
$$

and using the inequality (1.11) we have

$$
\int_{B_{r}}|\nabla w|^{4} \leq C\|w\|_{B M O\left(B_{r}\left(x_{0}\right)\right)}^{2} \int_{B_{r}}|\Delta \xi|^{2}
$$

we then obtain

$$
\frac{1}{\rho^{n-4}} \int_{B_{\rho}}|\nabla \xi|^{4} \leq C\left(\frac{\rho}{r}\right)^{4} \frac{1}{r^{n-4}} \int_{B_{r}}|\nabla \xi|^{4}+C k(n) \frac{1}{r^{n-4}} \int_{B_{r}}|\Delta \xi|^{2}
$$

Denote $T(r)=\frac{1}{r^{n-4}} \int_{B_{r}}|\nabla \xi|^{4}$, we have found $0<\lambda<1$ such that

$$
T(\lambda r) \leq \frac{1}{2} T(r)+C k(n) \frac{1}{r^{n-4}} \int_{B_{r}}|\Delta \xi|^{2}
$$

Iterating this identity we get that

$$
\sup _{x \in B_{1}, \rho<r<\lambda} \frac{1}{r^{n-4}} \int_{B_{r}}|\nabla \xi|^{4} \leq
$$

$$
\leq 2^{\log _{\lambda} \rho} \int_{B_{1}}|\nabla \xi|^{4}+C k(n) \sup _{x \in B_{1}, \rho<r<1} \frac{1}{r^{n-4}} \int_{B_{r}}|\Delta \xi|^{2}
$$


From (3.36) standard elliptic estimates imply

$$
\int_{B_{1}}\left|\nabla^{2} \xi\right|^{2} \leq C \int_{B_{1}}|\Delta \xi|^{2}
$$

and using (1.11) on $B_{1}$ we get

$$
\int_{B_{1}}|\nabla \xi|^{4} \leq k(n) \int_{B_{1}}\left|F_{A}\right|^{2}+|\nabla \xi|^{4}
$$

Combining (3.67) and (3.68) we obtain the desired result and Lemma 3.9 is proved.

Proof (of Theorem 1.3). Let $\tilde{A}$ and $\tilde{A}_{i}$ that approximates $\tilde{A}$. We apply Theorem 3.7 to $\tilde{A}_{i}$ and we get a Coulomb gauge $A_{i}$ on $B_{1}$ to which we apply the Lemma 3.9 above. Modulo extraction of a subsequence $A_{i}$ converges to some limiting $A$ which is in $M_{2}^{1,2}\left(B_{1}\right) \cap M_{1}^{0,4}\left(B_{1}\right)$. A solves of course $d^{*} A=0$ and $\iota_{\frac{\partial}{\partial r}} A=0$ on $\partial B_{1}$. Denote by $\sigma_{i}$ the change of gauge between $\tilde{A}_{i}$ and $A_{i}$. We have

$$
\sigma_{i} A_{i}=\tilde{A}_{i} \sigma_{i}+d \sigma_{i}
$$

From the convergences of $\tilde{A}_{i}$ and $A_{i}$ we deduce that $d \sigma_{i}$ is bounded in $L^{4}\left(B_{1}\right) \cap W^{1,2}\left(B_{1}\right)$. We may extract a subsequence such that $\sigma_{i}$ converges weakly in these spaces, which implies that it converges strongly in $L^{2}\left(B_{1}\right)$ and since both $\tilde{A}_{i}$ and $A_{i}$ converge strongly in $L^{2}$, the gauge equivalence equation passes to the limit and we get that $A$ and $\tilde{A}$ are gauge equivalent and Theorem 1.3 is proved.

\section{Proof of Theorem 1.1}

Theorem 1.1 will be a standard consequence of the epsilon regularity (see the covering arguments in [8]) Theorem 1.2. Moreover Theorem 1.2 is a consequence of the epsilon regularity for smooth Yang-Mills Fields established in [10] and [13] once we are able to prove that under the smallness assumption of the curvature density, gauge invariant quantities are smooth. Therefore Theorem 1.1 will be established once the following lemma will be proved.

Lemma 4.10 Let $D$ be a stationary Yang-Mills connection in $\mathfrak{M}_{2}^{1,2}\left(B_{1}\right) \cap$ $\mathfrak{M}_{1}^{0,4}\left(B_{1}\right)$. There exists $\kappa(n)$ independent on $D=d+\tilde{A}$ such that, assuming there exists a sequence of smooth $\tilde{A}_{i}$ converging strongly to $\tilde{A}$ in $L^{4} \cap W^{1,2}$ and such that $\int_{B_{1}}\left|F_{\tilde{A}_{i}}\right|^{2} \leq \kappa(n)$ then, if $A$ is the Coulomb gauge of $D=d+\tilde{A}$ given by Theorem 1.3 over $B_{\frac{1}{2}}$, $A$ is smooth on $B_{\frac{1}{2}}$. 
Proof (of Lemma 4.10). We will need the following lemma

Lemma 4.11 Let $f$ in $M_{3}^{0,4 / 3}\left(B_{1}\right)$ and $u$ solving

$$
\begin{cases}\Delta u=f & \text { in } B_{1} \\ u=0 & \text { on } \partial B_{1}\end{cases}
$$

we have

$$
\|u\|_{L^{4}\left(B_{1}\right)} \leq C\|f\|_{L^{\frac{4}{3}\left(B_{1}\right)}}^{1 / 3}\|f\|_{M_{3}^{0,4 / 3}\left(B_{1}\right)}^{2 / 3}
$$

Recall that

$$
\|f\|_{M_{3}^{0,4 / 3}\left(B_{1}\right)}^{4 / 3}=\sup _{r>0, x \in B_{1}} \frac{1}{r^{n-4}} \int_{B_{r}(x) \cap B_{1}} f^{\frac{4}{3}}
$$

Remark 4.3 The above lemma should certainly be well known from specialists but we prove it here for the convenience of the readers.

Proof (of Lemma 4.11). We adopt the notations of the proof of Lemma 3.5: $\phi(x)=x /|x|^{2}, \hat{g}_{i j}=\delta_{i j}$ in $B_{1}$ and $\hat{g}_{i j}=\delta_{i j} /|x|^{4}$ in $B_{2} \backslash B_{1}$. Denote also $\hat{u}=u$ in $B_{1}$ and $\hat{u}=-u \circ \phi$ in $B_{2} \backslash B_{1}$ finally $\hat{f}=f$ in $B_{1}$ and $\hat{f}=-f \circ \phi$ in $B_{2} \backslash B_{1}$. Clearly $\hat{u}$ solves $\Delta_{\hat{g}} \hat{u}=\hat{f}$ in $B_{2}$. Moreover it is not difficult to check that there exists a constant $C$, independent on $f$, such that

$$
\sup _{x \in B_{2}, r>0} \frac{1}{r^{n-4}} \int_{B_{r}(x) \cap B_{2}}|\hat{f}|^{\frac{4}{3}} \leq C \sup _{x \in B_{1}, r>0} \frac{1}{r^{n-4}} \int_{B_{r}(x) \cap B_{1}}|f|^{\frac{4}{3}}
$$

Denote $\tilde{f}$ the function equal to $\hat{f}$ in $B_{2}$ and equal to 0 in $\mathbb{R}^{n} \backslash B_{2}$. One interpolate smoothly $\hat{g}$ and $\delta_{i j}$ between $B_{2}$ and $B_{4}$ and keep denoting $\hat{g}$ the resulting metric on $\mathbb{R}^{n}$. Let $v=\Delta^{-1} \bar{f}$ on $\mathbb{R}^{n}$, where $\Delta^{-1}$ denotes the convolution with the Green Kernel of $\Delta_{\hat{g}}$. From proposition 3.1 of [1] we deduce

$$
\|v\|_{L^{4}\left(\mathbb{R}^{n}\right)} \leq C\|\bar{f}\|_{M_{3}^{0,4 / 3}\left(\mathbb{R}^{n}\right)}^{\frac{2}{3}}\|\bar{f}\|_{L^{\frac{4}{3}\left(\mathbb{R}^{n}\right)}}^{\frac{1}{3}}
$$

The harmonicity of $\hat{u}-v$ on $B_{2}$ implies

$$
\begin{aligned}
\|\hat{u}-v\|_{L^{4}\left(B_{1}\right)} & \leq C\|\hat{u}-v\|_{L^{4 / 3}\left(B_{2}\right)} \\
& \leq C\|f\|_{L^{4 / 3}\left(B_{1}\right)}+C\|v\|_{L^{4}\left(\mathbb{R}^{n}\right)}
\end{aligned}
$$

Combining (4.71) and (4.72) one gets Lemma 4.11. 
Proof (of Lemma 4.10). The assumption $\int_{B_{1}}\left|F_{D}\right|^{2} \leq \kappa(n)$ combined with the monotonicity formula implies

$$
\sup _{r<1 / 4 x \in B_{\frac{1}{2}}} \frac{1}{r^{n-4}} \int_{B_{r}(x)}\left|F_{D}\right|^{2} \leq \kappa(n)
$$

therefore we can apply Theorem 1.3 and we get the Coulomb gauge $A$ for $D$ that solves

$$
\left\{\begin{array}{l}
\Delta A=-d^{*}[A, A]-*\left[A, * F_{A}\right] \quad \text { in } B_{1 / 2} \\
\|A\|_{M_{1}^{0,4}\left(B_{1 / 2}\right)}^{2}+\|\nabla A\|_{M_{2}^{0,2}\left(B_{1 / 2}\right)}^{2} \leq C \kappa(n)
\end{array}\right.
$$

This yields in particular

$$
|\Delta| \leq C\left[|\nabla A|+|A|^{2}\right]|A| \quad \text { in } B_{1 / 2}
$$

Let $B_{r}(x) \subset B_{1 / 2}$ and $B$ solving

$$
\left\{\begin{array}{l}
\Delta B=-d^{*}[A, A]-*\left[A, * F_{A}\right] \quad \text { in } B_{r} \\
B=0 \quad \text { on } \partial B_{r}
\end{array}\right.
$$

In one hand, applying Lemma 4.11 and (4.74), we have

$$
\begin{aligned}
& {\left[\frac{1}{r^{n}} \int_{B_{r}(x)}|B|^{4}\right]^{1 / 4}} \\
& \leq C\left[\sup _{y \in B_{r}(x), r>0} \frac{1}{\rho^{n-4}} \int_{B_{\rho}(y) \cap B_{r}(x)}|\Delta B|^{\frac{4}{3}}\right]^{1 / 2}\left[\frac{1}{r^{n}} \int_{B_{r}}|\Delta B|^{\frac{4}{3}}\right]^{1 / 4} \\
& \leq C\left[\|A\|_{M_{1}^{0,4}\left(B_{1 / 2}\right)}^{2}+\|\nabla A\|_{M_{2}^{0,2}\left(B_{1 / 2}\right)}\right]\left[\frac{1}{r^{n}} \int_{B_{r}(x)}|A|^{4}+\frac{1}{r^{n}} \int_{B_{r}(x)}|\nabla A|^{2}\right]^{1 / 4}
\end{aligned}
$$

In the other hand, using the fact that $A-B$ is harmonic on $B_{r}(x)$, we have for any $\rho<r$

$$
\begin{aligned}
\int_{B_{\rho}}|A-B|^{4} & \leq C\left(\frac{\rho}{r}\right)^{n} \int_{B_{r}}|A-B|^{4} \\
\int_{B_{\rho}}|\nabla(A-B)|^{2} & \leq C\left(\frac{\rho}{r}\right)^{n} \int_{B_{r}}|\nabla(A-B)|^{2}
\end{aligned}
$$


A standard interpolation estimate applied to $B$ on $B_{r}(x)$ gives

$$
\frac{1}{r^{n-2}} \int_{B_{r}}|\nabla B|^{2} \leq C\left(\frac{1}{r^{n}} \int_{B_{r}}|B|^{4}\right)^{1 / 4}\left(\frac{1}{r^{n-8 / 3}} \int_{B_{r}}|\Delta B|^{\frac{4}{3}}\right)^{3 / 4}
$$

Therefore

$$
\frac{1}{r^{n-4}} \int_{B_{r}}|\nabla B|^{2} \leq C\left(\frac{1}{r^{n-4}} \int_{B_{r}}|B|^{4}\right)^{1 / 4}\left(\frac{1}{r^{n-4}} \int_{B_{r}}|A|^{4}+|\nabla A|^{2}\right)^{3 / 4}
$$

Combining (4.76), (4.77), (4.78) and (4.79) one gets

$$
\begin{aligned}
& \frac{1}{\rho^{n-4}} \int_{B_{\rho}}|A|^{4}+|\nabla A|^{2} \leq C\left(\frac{\rho}{r}\right)^{4} \frac{1}{r^{n-4}} \int_{B_{r}}|A|^{4}+|\nabla A|^{2} \\
& +C_{\rho / r}\left[\|A\|_{M_{1}^{0,4}\left(B_{1 / 2}\right)}^{8}+\|\nabla A\|_{M_{2}^{0,2}\left(B_{1 / 2}\right)}^{4}\right]\left[\frac{1}{r^{n-4}} \int_{B_{r}(x)}|A|^{4}+\frac{1}{r^{n-4}} \int_{B_{r}(x)}|\nabla A|^{2}\right]
\end{aligned}
$$

There exists $0<\lambda<1$ independent on $r$ such that $C \lambda^{4}<1 / 4$, assume now

$$
C_{\lambda}\left[\|A\|_{M_{1}^{0,4}\left(B_{1 / 2}\right)}^{8}+\|\nabla A\|_{M_{2}^{0,2}\left(B_{1 / 2}\right)}^{4}\right]<1 / 4
$$

and denote $T(r)=\frac{1}{r^{n-4}} \int_{B_{r}(x)}|A|^{4}+\frac{1}{r^{n-4}} \int_{B_{r}(x)}|\nabla A|^{2}$, then (4.80) becomes

$$
T(\lambda r) \leq \frac{1}{2} T(r)
$$

and we deduce the existence of $0<\alpha\left(\alpha=\left[\frac{\log (1 / 2)}{\log \lambda}\right]\right)$ such that

$$
\sup _{B_{r}(x) \subset B_{1 / 3}} \frac{1}{r^{n-4+\alpha}} \int_{B_{r}(x)}|A|^{4}+\frac{1}{r^{n-4+\alpha}} \int_{B_{r}(x)}|\nabla A|^{2}<+\infty
$$

This gain of regularity bootstraps as follows : of [1])

$$
\left\|M_{3-\delta} \Delta A\right\|_{L^{\infty}\left(B_{1 / 3}\right)}:=\sup _{B_{r}(x) \subset B_{1 / 3}} \frac{1}{r^{n-3+\delta}} \int_{B_{r}(x)}|\Delta A|<+\infty
$$

Therefore, for any $p>1$, we have from [1] proposition 3.1

$$
\|A\|_{L^{q}\left(B_{1 / 4}\right)} \leq C\left\|M_{3-\delta} \Delta A\right\|_{L^{\infty}\left(B_{1 / 3}\right)}^{2 p / \lambda}\|\Delta A\|_{L^{p}\left(B_{1 / 3}\right)}^{1-2 p / \lambda}+\|A\|_{L^{4}\left(B_{1 / 3}\right)}
$$


where $\lambda=p(3-\delta)$ and $q=p\left(\frac{3-\delta}{1-\delta}\right)$. We also have

$$
\|\nabla A\|_{L^{r}\left(B_{1 / 4}\right)} \leq C\left\|M_{3-\delta} \Delta A\right\|_{L^{\infty}\left(B_{1 / 3}\right)}^{p / \lambda}\|\Delta A\|_{L^{p}\left(B_{1 / 3}\right)}^{1-p / \lambda}+\|A\|_{L^{4}\left(B_{1 / 3}\right)}
$$

where $r=p\left(\frac{3-\delta}{2-\delta}\right)$. Observe that $|\Delta A| \leq C|A|^{3}+|\nabla A|^{3 / 2}$, thus we have

$$
\|\Delta A\|_{L^{p}} \leq C\|A\|_{L^{3 p}}^{3}+\|\nabla A\|_{L^{3 p / 2}}^{3 / 2} .
$$

Using the fact that $\frac{3-\delta}{1-\delta}>3$ and $\frac{3-\delta}{2-\delta}>\frac{3}{2}$ ( since $\delta>0$ ), we have that $q-3 p$ admits a fixed positive lower bound for $p \geq 4 / 3$, so as $r-3 p / 2$. Thus, modulo an eventual reduction of the size of the ball, one bootstraps $L^{p}$ estimates for $A$ and $\nabla A$ until reaching for instance that $\Delta A \in L^{q}$ for $q>n$ which implies that $F_{A} \in L^{\infty}$ and standard Sobolev Bootstraping in equation (4.73) tells us that $A$ is analytic in a ball of fixed radius (that could be $B_{1 / 2}$ modulo a reduction of $\kappa(n))$.

Once this work was completed we learned that independently Terence Tao and Gang Tian established the existence of Coulomb Gauges in Morrey Spaces and proved a similar regularity result for stationary Yang-Mills called "admissible" by the authors (i.e. stationary Yang-Mills Fields which are weakly approximable by smooth Yang-Mills Fields) — see [12].

\section{References}

[1] Adams, D. R.: A note on Riesz Potentials. Duke Math J. 42 (1975), $765-778$.

[2] Bourguignon, J. P., Lawson, H. B. JR.: Yang-Mills Theory : its physical origins and differential geometry aspects. In Seminar on differential Geometry, S. T. Yau, ed. Annals of Mathematics Studies 102, Princeton University Press, 1982, 395-422.

[3] Brezis, H.: Analyse fonctionnelle, Masson, Paris, 1983.

[4] Donaldson, S. K., Kronheimer, P. B.: The geometry of FourManifolds. Oxford, 1990.

[5] Evans, L. C.: Partial regularity for stationary harmonic maps into spheres. Arch. Ration. Mech. Anal. 116 (1991), 101-113.

[6] Cohen, A., Meyer, Y., Oru, F.: Improved Sobolev embedding. Sémin. Équ. Dériv. Partielles, 1997-1998, no. XVI. École Polytechnique, 1998.

[7] Giaquinta, M.: Multiple integrals in the calculus of variations and non-linear elliptic systems. Ann. of Math. Stud. 105, Princeton Univ. Press, 1983. 
[8] HÉlein, F.: Harmonic maps, Conservation laws and moving frames. Diderot, 1996.

[9] Morrey, C. B.: Multiple integrals in the calculus of variations. Springer, New York, 1966.

[10] Nakajima, H.: Compactness of the moduli space of Yang-Mills connections in higher dimensions. J. Math. Soc. Japan 40 (1988), 383-392.

[11] Price, P.: A Monotonicity Formula for Yang-Mills Fields. Manuscripta Math. 43 (1983), 131-166.

[12] TaO, T., Tian, G.: A singularity removal theorem for Yang-Mills fields in higher dimensions, in preparation.

[13] Tian, G.: Gauge theory and calibrated geometry, I. Ann. Math. 151 (2000), 193-268.

[14] Uhlenbeck, K.: Connections with $L^{p}$ bounds on curvature. Comm. Math. Phys. 83 (1982), 31-42.

Recibido: 11 de septiembre de 2001

\section{Yves Meyer \\ CMLA, ENS-CACHAN}

61, av. du Président Wilson

94235 Cachan Cedex, France ymeyer@cmla.ens-cachan.fr

Tristan Rivière

Centre de Mathématiques

Ecole Polytechnique

91128 Palaiseau Cedex, France riviere@math.polytechnique.fr 